\section{P41 Yumbox: Bringing MyPlate to Preschoolers' Lunches}

JenniferShukaitis, MPH, shukaitis@njaes.rutgers.edu, Rutgers University, 88 Lipman Dr, Martin Hall, Room 301, New Brunswick, NJ, 08901; Sara Elnakib, PhD, RDN, Rutgers University; Cuite Cara, PhD, Rutgers University

Objective: To test whether Yumbox, a bento-style lunchbox with compartments labeled for the 5 meal components of USDA's MyPlate, has an effect on foods that caregivers packed for preschoolers' lunches.

Use of Theory: Yumbox provides distinct compartments for recommended food components of USDA MyPlate, leveraging visibility principals of behavioral economics theory. Caregivers may be more likely to pack lunches that include more components of MyPlate due to visual cues present in Yumbox.

Target Audience: Caregivers of preschoolers

Program Description: Yumboxes were given to each child in a preschool ("Intervention School") $(\mathrm{n}=30)$. Caregivers received a brief orientation on using Yumbox, as well as a list of suggestions for foods for each section. A similar preschool was selected for comparison of the content of students' lunches ("Control School"; $n=26$ ). Contents of the students' lunches in both preschools were documented over a 3-day period by photographing lunches in both schools ( $\mathrm{N}=110$ lunches). Photos were then analyzed to identify if a meal component was present or absent in the students' lunches.

Evaluation Methods: All data were entered into SPSS for analysis. Descriptive statistics of food present for each component (vegetable, fruit, dairy, grain, protein), for both the Intervention and Control Schools were conducted. Chi-square analyses were also conducted to assess significant group differences in the presence of each food component. A comparison of the mean number of food components present on each student's trays were used to assess impact of the intervention.

Results: This study found that the intervention group had had statistically significantly higher number of components $(3.78 \pm 0.95)$ compared to the control group $(1.89 \pm 0.93) t=-10.56 ; P<0.0001$. Chi-square tests found significant differences across all days for fruit, protein, and dairy, and significant differences on the first 2 days for vegetables.

Conclusion: This study provides evidence that Yumbox may have a positive influence on the types of foods caregivers pack for children's lunches.

Funding: None.

\section{Food and Nutrition Policy}

\section{P42 A Longitudinal Analysis of School Garden Prevalence Across 4 New Jersey Cities}

Naomi Reyes, BS, ngreyes1@asu.edu, Arizona State University, 411 North Central Ave, Phoenix, AZ, 85004; Punam Ohri-Vachaspati, PhD, RDN, Arizona State University
Background: School gardens are a popular strategy to increase preference and consumption of fruits and vegetables (FV). The Healthy Hunger Free Kids Act (HHFKA) of 2010 supported implementation of school gardens for promoting FV consumption. Current research lacks longitudinal assessments of changes in prevalence of school gardens over time.

Objective: In this study, we examine the prevalence of school gardens over time by school level factors before and after the implementation of the Healthy Hunger Free Kids Act (HHFKA).

Study Design: We used data from the New Jersey Child Health Study (NJCHS) conducted in 4 low-income NJ cities. Information on school garden prevalence was collected from all schools $(n=148)$ in the 4 study cities for school years between 2010-11 and 2017-18.

Analysis: Prevalence of school gardens was calculated for each school year over the study period. Multivariable analysis estimated changes in prevalence of school gardens over time adjusting for school-level factors including, school level (elementary vs middle/high), enrolled students' race/ethnicity, and enrolled students' eligibility for free and reduced-price meals (FRPM).

Results: There were 97 elementary and 51 middle/high schools in the sample. In 2010-11, 19\% of all K-12 schools participated in school gardens. Multivariate analysis showed that after adjusting for school-level factors, compared to 2010-11, a higher proportion of schools reported having a garden in 2013-14 (29\%, $P=0.055), 2014-15$ (31\%, $P=0.018)$, and in 2016-17 (31\%, $P=0.051)$. Schools with majority Hispanic student enrollment had less than half the odds of having a garden compared to schools with majority Black students $(P=0.045)$. There were no differences in garden prevalence between elementary and middle/high schools or FRPM eligibility.

Conclusion: School garden presence showed patterns of increasing prevalence over time, especially in the years following the HHFKA. Future research should examine the reasons for differences in prevalence of gardens in schools with racially and ethnically diverse student populations over multiple time points.

Funding: Robert Wood Johnson Foundation, Eunice Kennedy Shriver National Institute of Child Health and Human Development National Institutes of Health (1R01HD071583-01A1), and the National Heart, Lung, and Blood Institute, National Institutes of Health (1R01HL137814-01).

\section{P43 Conceptual Model to Assess the Resilience Capacity of School Meal Programs: A Systematic Review and Thematic Analysis}

Kritika Gupta, MS, University of Mississippi; Georgianna Mann,PhD, grmann@olemiss.edu, University of Mississippi, 220 Lenoir Hall, Sorority Row, PO Box 1848, 


\section{P43 (continued)}

University, MS, 38677; Laurel Lambert, PhD, $R D$, University of Mississippi

Background: Unprecedented school closures during the COVID-19 pandemic brought into question the resilience capacity of school meal programs. Resilience capacity is defined as the capacity to endure shocks and stressors including absorptive capacity (persistence), adaptive capacity (incremental adjustment), and transformative capacity (change). Resilience Capacity Model assesses the resilience capacity of a system based on the actors and corresponding components of that system. Resilience capacity has been explored in school nutrition setting but a Resiliency Capacity Model addressing school meal provision is lacking.

Objective: Qualitative exploration of a Resilience Capacity Model to assess the resilience capacity of school meal programs.

Study Design, Settings, Participants: Assisted by PRISMA, a systematic evaluation of peer-reviewed full-text articles published between 2005-2020 was performed.

Measurable Outcome/Analysis: Qualitative and quantitative studies explored the effect of school meal programs or in-school interventions on child food security that accounts for resilience capacity of school meal programs. Thematic analysis was used to identify factors associated with strategies for building resilience capacity.

Results: Of 406 articles identified via online search, 32 full-text articles met inclusion criteria. Based on the systematic review and thematic analysis, it was found that strategies to build resilience include food availability, food accessibility, food quality, education, training, parent/ teacher support, and administrative response. The Resilience Capacity Model to assess resilience capacity of school meal programs fits well in the context of school nutrition. This model can be used to assess whether the strategies adopted during unprecedented circumstances lead to a resilience or a vulnerability pathway.

Conclusion: The findings of the systematic review of literature support that Resilience Capacity Model that accounts for identified factors can be used to assess the resilience capacity of school meal programs. A systematic assessment of resilience capacity can help to improve school meal program operations in times of crisis or during/after disasters.

Funding: None.

\section{P44 Costing a Sustainable, Basic 7-Day Menu: A Tool for Policymakers and Educators to Help Ensure Adequacy in Dietary Intake}

Suzanne Piscopo, PhD, MA, B.Ed, R.Nutr, R. Eur Health Promotion Practitioner, suzanne.piscopo@um.edu.mt, University of Malta, Faculty of Education, Dept. of Health, Physical Education and Consumer Studies, Tal-Qroqq, Msida, Malta, MSD2080; Andre Bonello, MA, BA, Caritas Malta, Head of Research and Advocacy; Anthony Gatt, MA, B.Psy, Caritas Malta, Director
Background: Caritas Malta is a not-for-profit organisation which strives to eradicate poverty and promote a decent quality of life for the most vulnerable. Ensuring an accessible, appropriate, nutritious diet is 1 of its activities.

Objective: : A study was conducted by Caritas Malta in 2019-20 to establish A Minimum Essential Budget for a Decent Living (MEBDL), covering basic necessities for lowincome households. This included the amount required for food. The goals were to offer evidence for food security policy development, and to produce a tool for counselling on food budgeting and meal planning.

Study Design, Settings, Participants: Three 7-day menus were created based on the Maltese national dietary guidelines, incorporating traditional dishes, whilst taking into account contemporary lifestyles. The menus included 3 meals and 2 snacks daily and met the nutritional needs of 3 configurations of low-income households ( 2 adults and 2 children, 1 adult and 2 children, 2 elderly persons). Feedback on the menus was obtained from representatives of these target households during 1-to-1 or focus group interviews.

Measurable Outcome/Analysis: The 3 7-day menus were costed in a supermarket having moderate prices. Local, fresh, healthy ingredients were opted for as far as possible. The cost of food aid packages distributed to low-income households as part of national food security programmes was deducted from each of the 3 menu totals.

Results: The monthly cost of the 3 menus were $€ 593.45$, $€ 430.65$ and $€ 281.24$ for the households comprising 2 adults and 2 children, 1 adult and 2 children, and 2 elderly persons, respectively. This food cost corresponded to $41 \%$ to $51 \%$ of the total MEBDL. When the cost of eating out once a month was added (simple meal or snacks) this increased the cost by $€ 10$ per person.

Conclusion: In low-income households, food accounts for a high share of total expenses. Nutrition educators have a key role in providing guidance to policymakers, and advice to households, on maximising nutrient density in dietary intake based on income.

Funding: None.

\section{P45 Do "Natural" and Other Clean Labels Detract from more Regulated Food Labels?}

Nick Rose, MS, nick.rose@uvm.edu, University of Vermont, 31 Nash Pl, Burlington, VT, 05401; Travis Reynolds, PhD, University of Vermont; Jane Kolodinsky, PhD, University of Vermont

Background: "Clean labeling" is a food industry trend characterized by simpler ingredients and front of package marketing statements that attract health-oriented consumers looking for more natural foods. Many clean labels, including "all natural" are not clearly defined, nor regulated, and have the potential to detract from food labels that are regulated by the FDA and/or USDA.

Objective: To compare consumer use of federally regulated (organic) and unregulated (natural) food labels, and to determine whether use of these labels is associated with demographic characteristics. 